\title{
Concealed Wenckebach phenomena in the left bundle-branch
}

\author{
H. David Friedberg and Leo Schamroth
}

From The Cardiovascular Section, Veterans Administration Center, Wood (Milwaukee); St. Luke's Hospital; and the Medical College of Wisconsin (formerly Marquette School of Medicine), Milwaukee, Wisconsin; and Baragwanath Hospital and the University of the Witwatersrand, Fohannesburg, South Africa

Two cases of intermittent left bundle-branch block are presented and interpreted as reflecting Wenckebach sequences, confined to the left bundle-branch, which are incompletely concealed. Published reports of clinical, experimental, and His bundle studies are reviewed. The possible variations are analysed, and criteria are given for the diagnosis from clinical electrocardiograms. These cases present further evidence that the Wenckebach phenomenon (or Mobitz Type I block) may occur anywhere in the heart, and is not necessarily confined to the AV node proper.

Clinical electrocardiograms demonstrating Wenckebach conduction confined to a bundlebranch are rare. Four cases have been reported (Vitolo and Rossi, 1961; Rosenbaum et al., 1969; Friedberg and Schamroth, 1969. In the case of Katz and Pick (1956), the Wenckebach phenomenon occurred in both branches with different conduction ratios in each. Rosenbaum et al. (1969) have delineated 'direct' and 'indirect' Wenckebach series in a bundle-branch. This paper presents two further examples of indirect Wenckebach sequences confined to the left bundle-branch. Since the phenomenon is not directly visible in the electrocardiogram but is inferred, it may be spoken of as 'concealed'.

\section{Case reports}

Case I The electrocardiogram (Fig. I) was recorded from a 57-year-old man with calcific aortic stenosis, shortly after surgical replacement of the aortic valve. There is regular sinus rhythm: the PP intervals are constant and measured 0.66 second, reflecting a rate of 91 beats per minute. There is first degree AV block: the PR intervals measure 0.31 second and do not vary. There are two forms of QRS complex which are distributed in a definite rhythmic pattern and labelled $I$ to 5 (standard lead II). Four consecutive beats - numbered 2 to 5 - show complete left bundle-branch block (QRS duration =0.16 sec). These are followed by a beat (labelled $\mathbf{I}$ ) which is more normally conducted (QRS duration $=0.12 \mathrm{sec}$ ). This beat shows an increased ventricular activation time $(0.05 \mathrm{sec})$ - a reflection of left ventricular hypertrophy. There is a small terminal QRS vector

Received 30 June 197 I. directed inferiorly, slightly anteriorly, and to the right - a manifestation of incomplete right bundlebranch block. There are primary ST segment and $\mathrm{T}$ wave changes.

Case 2 The electrocardiograms (Fig. 2) were recorded from a 60-year-old man with coronary artery disease and consist of a section of standard lead II and two selected nearly contiguous strips of lead aVR. There is regular sinus tachycardia. The PP intervals are constant and measure 0.5 second, reflecting a rate of 120 beats per minute. There is a first degree AV block (PR intervals =

FIG. I Case I Partly concealed 5:4 block in the left bundle-branch, with the Wenckebach phenomenon and constant incomplete right bundle-branch block. Electrocardiograms, selected leads, showing a regular succession of four beats with complete left bundle-branch block (labelled 2 to 5) followed by one with incomplete right bundle-branch block (labelled I). See text.

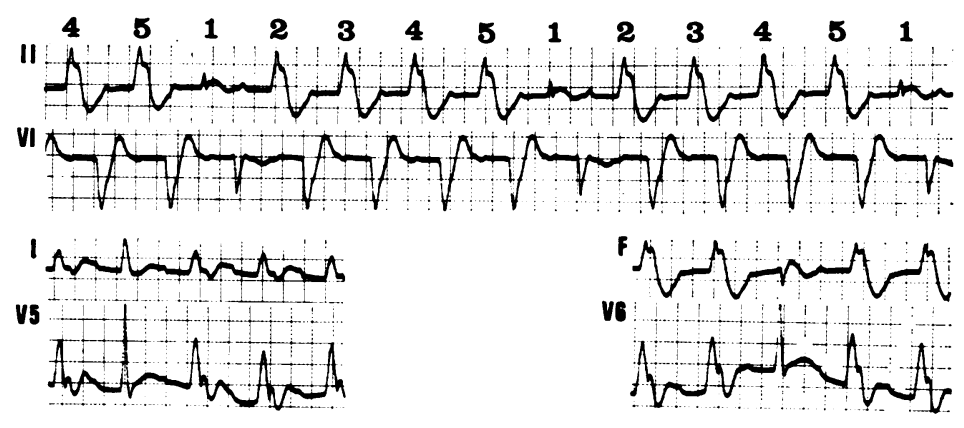


$0.36 \mathrm{sec}$, and do not vary). In standard lead II, pairs of beats with complete left bundle-branch block (QRS duration $=0.15 \mathrm{sec}$ ) are followed by a third beat with incomplete left bundle-branch block ( $Q R S$ duration $=0 \cdot 10 \mathrm{sec}$ ). In lead aVR, the sequences vary somewhat: in the upper strip there is a run of 3 , and in the lower strip a run of 4 successive beats with complete left bundlebranch block.

\section{Discussion}

When the supraventricular rhythm is irregular, intermittent bundle-branch block may be an expression of phasic aberrant ventricular conduction. Under this circumstance, a relatively early supraventricular impulse encounters an incompletely recovered bundle-branch, giving rise to a bundle-branch block pattern: a relatively late impulse finds both bundlebranches responsive and is consequently normally conducted. When the supraventricular rhythm is strictly regular, as in the cases presented here, this mechanism cannot apply. A true second-degree block within a bundlebranch must be postulated.

If conduction in one bundle-branch is delayed by more than at most 0.06 second, the ventricle will be activated from the contralateral bundle-branch (Rosenbaum and Lepeschkin, 1955; Katz and Pick, 1963). ${ }^{1}$ The electrocardiographic appearance of complete bundle-branch block results, even though there is only a delay in conduction and complete block is not present in that branch. Any additional conduction delay in the affected branch will not further affect the QRS-T complex. If - in any beat - the bundle-branch is not activated because it is refractory, the prolonged rest period so afforded may allow improved conduction in the next beat. The variations in intraventricular conduction during regular rhythm in these 2 cases may then be explained as follows.

In the beats numbered $I$ in Fig. $I$, conduction occurs down both bundle-branches with a fractionally greater delay in the right bundlebranch. In the beats numbered 2, 3, and 4 there is a delay or block within the left bundlebranch. Conduction through the left bundle reasumes in each beat $\mathrm{I}$. It follows, therefore, that in the immediately preceding beat (beat 5) there must have been a complete block (and not merely a delay) in order to allow the left bundle an adequate time to recover. Therefore, in all beats labelled 5 , conduction in the left bundle is completely blocked.

\footnotetext{
1 Recent work using His bundle and conducting system electrograms suggests that this critical interval may be as small as 0.025 second (Narula and Samet, I970).
}

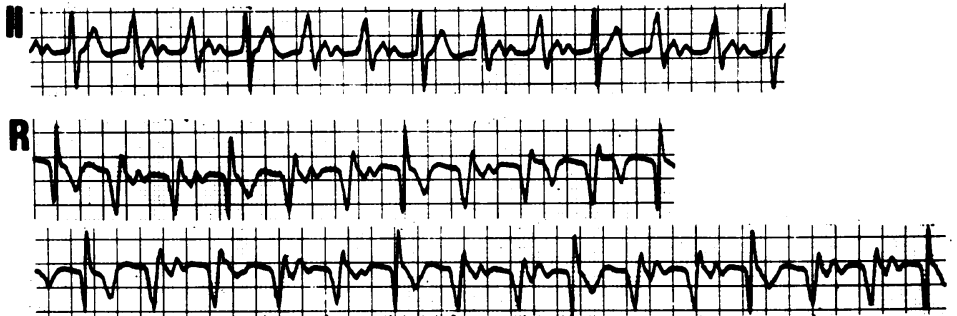

FIG. 2 Case 2. Electrocardiograms, standard lead II and two nearly continuous sections of lead aVR showing partly concealed varying left bundle-branch block with the Wenckebach phenomenon. The conduction ratio is $3: 2$ in standard lead II and in most of leads aVR. At the end of the upper strip of lead aVR the ratio changes to $4: 3 ;$ in the beginning of the lower strip it is 5:4.

Beats 2, 3, and 4 could also conceivably reflect complete block with the left bundlebranch. This would then represent a regular 5:I left bundle-branch block. This, however, is unlikely for the following reasons: (I) $5: 1$ conduction ratios are very uncommon in any form of second-degree block. Concealed conduction of alternate impulses usually results in even numbered conduction ratios. (2) Regularly repeating 5: I conduction ratios are even rarer. (3) This further implies that the refrac-

FIG. 3 Diagram to show the conduction variations in Fig. I. $A=$ atrial level; $A V=$ $A V$ junction; $B B=$ bundle-branches (labelled $R$ and $L) ;$ and $V=$ ventricular level. The numbers correspond to the beats so labelled in Fig. I. In beat I, left bundle-branch conduction is relatively normal, while that in the right is a little slowed. In beats 2, 3, and 4, progressively slower conduction occurs in the left bundle; the ventricles are activated from the right bundle-branch. In beat 5, conduction fails entirely within the left bundle, allowing recovery in the next beat $I$. The cycle then repeats.

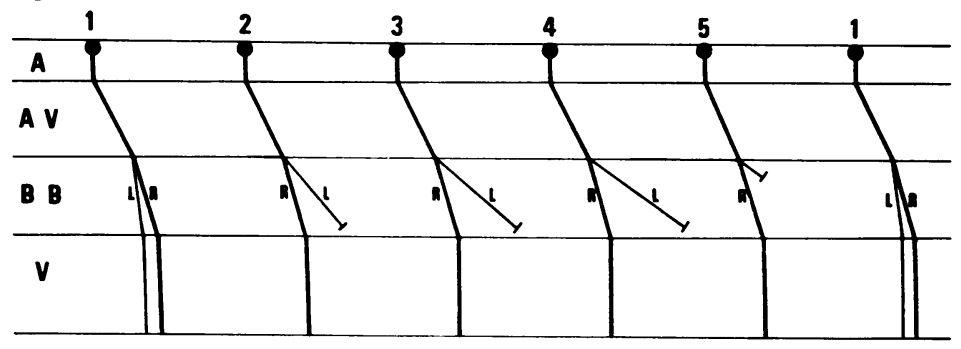


tory period of the left bundle extends over 4 cardiac cycles.

A more likely explanation for the manifestation of intermittent bundle-branch block in this case is a Wenckebach form of concealed conduction within the left bundle-branch. The hypothesis is illustrated diagrammatically in Fig. 3. Sinus impulses equivalent to beats I to 5 , in Fig. I, are similarly labelled. Sinus impulse $I$ is conducted through both bundlebranches with a minimal relative delay in the right bundle-branch. The conduction of sinus impulse 2 suffers delay within the left bundlebranch. The delay permits the left bundlebranch distal to the region of delay and the left ventricle to be activated entirely by the impulse conducted through the right bundlebranch. This results in a pattern of complete left bundle-branch block. Sinus impulses 3 and 4 suffer greater delays in conduction, that is, they are associated with further increments in conduction time. As the left bundle-branch 'block' is already maximal, these further delays have no effect on the left bundle-branch block pattern. Sinus impulse 5 is truly blocked, that is it fails to enter the zone of depressed conduction within the left bundlebranch which consequently has an opportunity to recover so that the next sinus impulse sinus impulse $I$ of the ensuing sequence - is conducted down both the bundle-branches once again. The sequence is then repeated. Progressive delay followed by failure, and then improvement of conduction is the Wenckebach phenomenon. Thus, Case I may be interpreted as showing second-degree block confined to the left bundle-branch with the Wenckebach phenomenon, the conduction ratio being $5: 4$.

Case 2 may be interpreted in a similar manner. Standard lead II and parts of lead aVR reflect 3:2 block in the left bundlebranch with the Wenckebach phenomenon. In the remainder of lead aVR, the conduction ratio changes momentarily to 4:3 (upper strip) or to 5:4 (lower strip). The alternative explanation involving $3: I, 4: I$, or $5: I$ left bundle-branch block is much less likely for the reasons given above.

Several further inferences may be drawn.

I) The PR intervals are constant in both cases. Conduction times in the right bundlebranch, therefore, do not vary.

2) In beat $I$ of Case $I$, the pattern of incomplete right bundle-branch block is seen. Left bundle-branch conduction in this beat must be slightly better than in the right, though it is considerably worse in later beats of the sequence. The PR intervals do not vary

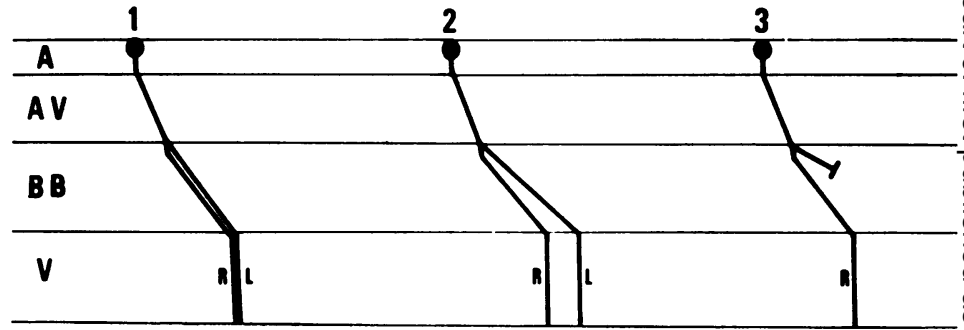

FIG. 4 Diagram to show a fully manifest Wenckebach sequence. Conventions as in Fig. 3. In beat 2 , the delay in left bundle-branch conduction is not as conspicuous as in beat 2 of Fig. 3; the pattern of incomplete left bundle-branch block results.

despite the conduction delays in both bundlebranches. It follows that the site of conduction delay accounting for the incomplete right bundle-branch block is not in the initial portion of the right bundle. It is situated distal to the fibres through which the left ventricle is activated.

3) There can be no antidromic or retrograde invasion of the left bundle-branch from the right side in the last beat of the Wenckebach sequence. Late retrograde activation of the bundle would reset its refractory period and destroy the Wenckebach sequence.

4) According to Rosenbaum (1969) and Rosenbaum, Elizari, and Lazzari (I970), left bundle-branch block may be the result of block of the main trunk (predivisional), or of block of both the anterior and posterior divisions (divisional left bundle-branch block). These two forms cannot ordinarily be separated. In these two cases, however, one may confidently infer that the block is predivisional. The alternative possibility, that there is a Wenckebach phenomenon in both divisions with the same conduction ratio, and, even when this ratio changes, the two divisions never get out of step, is too remote.

Monofascicular sequences Puech et al. (1970) have described a case of 3:2 AV block, right bundle-branch block, and left anterior hemiblock. His bundle electrograms showed the Wenckebach phenomenon occurring distal to the His deflection. A trifascicular system has been transformed, in effect, to a monofascicular system: the Wenckebach phenomenon is occurring in either the distal His bundle, the main left bundle, or in the posterior division. This is the clinical analogue of earlier experimental work of Scherf and Shookhoff (1925). 
Manifest and concealed sequences $A$ Wenckebach phenomenon in a bundle-branch may be fully manifest, or partly or completely concealed. If each successive beat of a series shows increasing bundle-branch block (Fig. 4), the sequence is fully manifest or 'direct'. Once the conduction delay in the affected bundle exceeds the time required for activation from the contralateral bundle, all later beats in the series will show complete bundlebranch block, and the latter part of the sequence will be partly concealed. If circumstances are such that the first beat is 'normally' conducted and the second shows complete bundle-branch block, as in these cases, the partly concealed sequence is termed by Rosenbaum 'indirect'. In the cases published by Rosenbaum and colleagues (I969), both manifest and partly concealed sequences occurred in the same record, demonstrating their very close relation.

Completely concealed sequences. If the first beat of a Wenckebach sequence has so much delay that a complete bundle-branch block pattern results, a Wenckebach phenomenon may well be there, but since all beats have the same shape it would be completely concealed. It could not be diagnosed, but may be suspected if a decrease in rate permitted a manifest or partly concealed sequence to result (Friedberg and Schamroth, 1969).

Diagnosis from clinical electrocardiograms Wenckebach conduction in a bundle-branch may be diagnosed under the following circumstances.

I) There is a strictly regular sinus (or other supraventricular) rhythm.

2) AV conduction time ( $P R$ interval) is strictly regular.

*3) QRS complexes with a more normal contour appear periodically.

4) If successive QRS complexes show progressively increasing bundle-branch block, a manifest Wenckebach sequence is diagnosed.

5) If all beats but the first show a complete block pattern, an incompletely concealed sequence is inferred.

Clinical significance There is, as shown above, evidence from clinical electrocardiograms, intracardiac tracings, and animal experiments to show that the Wenckebach phenomenon can occur in the bundle of His or in its branches. It seems most likely that the Wenckebach phenomenon is a fundamental property of any cardiac conducting tissue. The evidence does not support the opinion that this is a property only of the AV node (Watanabe and Dreifus, 1966), or that such periods in the sinoatrial junction are improbable (Scherf, 1969). Nor does this evidence support the concept that therapeutic decisions about pacing may be predicated entirely on the supposition that Wenckebach (Type I) $\mathrm{AV}$ block is confined to the AV node.

\section{References}

Friedberg, H. D., and Schamroth, L. (1969). The Wenckebach phenomenon in left bundle-branch block. American fournal of Cardiology, 24, 591.

Katz, A. M., and Pick, A. (1963) Transseptal conduction time in the human heart: evaluation of fusion beats in ventricular parasystole. Circulation, 27, I06I.

Katz, L. N., and Pick, A. (1956). Clinical Electrocardiography. Part I. The Arrhythmias, p. 624. Lea \& Febiger, Philadelphia.

Narula, O. S., and Samet, P. (1970). Wenckebach and Mobitz type II A-V block due to block within the His bundle and bundle-branches. Circulation, 41, 947.

Puech, P., Grolleau, R., Latour, H., Dufoix, R., Cabasson, J., and Robin, J. (1970). L'enregistrement de l'activité électrique du faisceau de $\mathrm{His}$ dans les blocs A.-V. spontanés. Archives des Maladies du Coeur et des Vaisseaux, 63, 784.

Rosenbaum, M. B. (1969). Types of left bundle-branch block and their clinical significance. Fournal of Electrocardiology, 2, 197.

Rosenbaum, M. B., Elizari, M. V., and Lazzari, J. O. (1970). The Hemiblocks, p. 157. Tampa Tracings, Oldsmar, Florida.

Rosenbaum, M. B., and Lepeschkin, E. (1955). Bilateral bundle-branch block. American Heart Fournal, 50, 38.

Rosenbaum, M. B., Nau, G. J., Levi, R. J., Halpern, M. S., Elizari, M. V., and Lazzari, J. O. (1969). Wenckebach periods in the bundle-branches. Circulation, 40, 79.

Scherf, D. (1969). The mechanism of sinoatrial block. American fournal of Cardiology, 23, 769 .

Scherf, D., and Shookhoff, C. (1925). Reizleitungstorungen im bundel: II. Mitteilung. Wiener Archiv für innere Medizin und deren Grenzgebiete, 11, 425.

Vitolo, E., and Rossi, L. (I96I). Blocco di branca sinistro con allungamento progressivo della conduzione e fenomeno di Wenkebach ventricolare. Minerva Cardioangiologica, 9, 254.

Watanabe, Y., and Dreifus, L. S. (1966). Second degree atrioventricular block. In Mechanisms and Therapy of Cardiac Arrhythmias, p. 448. Ed. by L. S. Dreifus, W. Likoff, and J. H. Moyer. Grune \& Stratton, New York.

Requests for reprints to Dr. H. David Friedberg, Cardiovascular Section - IIIA, Veterans Administration Center, 5000 West National Avenue, Wood (Milwaukee), Wisconsin 53193, U.S.A. 